\title{
EL RUIDO Y EL DISEÑO DE UN AMBIENTE ACÚSTICO
}

(1) María Párraga Velásquez

(2) Teonila García Zapata

\section{RESUMEN \\ El presente trabajo muestra conceptos generales sobre el ruido y sus efectos en las personas y propone recomendaciones para el diseño de un ambiente acústico adecuado y cómodo para los trabajadores. \\ Palabras Claves: Sonido, ruido, ambiente acústico, comodidad.}

NOISE AND THE DESIGN OF AN ACOUSTIC ENVIRONMENT

ABSTRACT

This study presents general concepts about noise and its effects on people. It also makes suggestions to design an appropriate and comfortable acoustic environment to workers.

Key words: Sound, noise, acoustic environment, comfort.

(1) Ingeniero Industrial. Profesor del Departamento de Gestión y Producción Industrial, UNMSM. E-mail: mparragav@unmsm.edu.pe

(2) Magíster en Administración Pública. Profesor del Departamento de Gestión y Producción Industrial,UNMSM. E-mail:teogaza57@yahoo.es

\section{INTRODUCCIÓN}

El ruido no es exclusivo de los ambientes de trabajo y, en nuestro país, lo encontramos por todas partes, debido a una falta de planificación urbana y de control en el cumplimiento de las normas. El ruido proviene de muchas fuentes y, en esta ocasión, nos ocuparemos del ruido en el ambiente laboral.

El ruido es un agente contaminante de tipo físico, estudiado tanto por la higiene industrial como por la ergonomía; sin embargo, el objeto de estudio de cada una de éstas es diferente. En cuanto a la primera, se preocupa por evitar la adquisición de una enfermedad profesional suscitada por la exposición del trabajador a agentes contaminantes, la segunda, busca un equilibrio entre el hombre y su entorno laboral, procurando brindar una situación de comodidad en el trabajo.

En este trabajo se tratará el ruido desde un punto de vista ergonómico.

\section{EL RUIDO}

El ruido es un sonido no deseado y, por lo tanto, incómodo. El ruido se define como el sonido o grupo de sonidos de tal amplitud que produce molestia o interferencia en la comunicación. La diferencia entre sonido y ruido radica en que el primero puede ser cuantificado, mientras que el segundo es un fenómeno subjetivo.

La medición de sonidos es a través de sonómetros y de dosímetros. Para que los resultados de la medida del sonido se parezcan lo más que se pueda a la percepción del oído humano, los instrumentos de medida llevan incorporados filtros o redes de compensación que determinan las escalas $A, B, C$ o D. La más utilizada es la escala $A$, por ello, los resultados de ruido industrial se dan en decibelios $A[\mathrm{~dB}(A)]$.

Existen diferentes criterios para indicar límites permisibles al ruido. El criterio de la ACGIH fija el límite o TLV en $85 \mathrm{~dB}(\mathrm{~A})$ para 8 horas de trabajo diario. El criterio ISO en su normativa que fija el límite en $90 \mathrm{~dB}(\mathrm{~A})$ para semanas de 40 horas. La diferencia entre estos dos criterios se encuentra en el factor de equivalencia (ver Cuadro 1). En cada criterio, se establece que el ruido se reducirá a la mitad del tiempo de exposición, si el valor medido se excede con relación a la norma en 3 ó $5 \mathrm{~dB}$, según corresponda (Factor de equivalencia). 
Cuadro 1. Tiempos de exposición al ruido

\begin{tabular}{|c|c|}
\hline Criterio ACGIH & Criterio ISO \\
\hline Factor de equivalencia $=5$ & Factor de equivalencia $=3$ \\
\hline$T=\frac{16}{2^{(N-80) / 5}}$ & $T=\frac{16}{2^{(N-87) / 3}}$ \\
\hline $\mathrm{N}=$ nivel sonoro en dB (A); $\mathrm{T}=$ tiempo en horas \\
\hline
\end{tabular}

\section{EFECTOS DEL RUIDO EN EL DESARROLLO DE LAS TAREAS}

Existen situaciones en las cuales el oído del trabajador se daña por estar expuesto a niveles de ruido perjudiciales, sufriendo lesiones, tales como el trauma acústico agudo y la sordera profesional. Sin embargo, también se presentan otras alteraciones no auditivas como las fisiológicas y/o las psíquicas derivadas del ruido.

Entre los efectos fisiológicos, se puede mencionar el aumento de la tensión vascular cerebral y la disminución de la capacidad motriz e intelectual, con el consiguiente aumento de errores en trabajos de precisión, aumento de la tensión arterial, estrechamiento del campo visual y modificación de los colores percibidos, etc. Los efectos psíquicos se centran básicamente en tres aspectos: El estado de ánimo, la molestia y la efectividad, dado que el trabajador deberá aumentar su nivel de concentración para llevar a cabo su tarea, lo cual provocará un incremento de la fatiga.

\section{DISEÑO DEL AMBIENTE ACÚSTICO}

El ruido es un problema en los ambientes de trabajo. Se presenta, tanto en talleres como en oficinas. Las fuentes que lo originan pueden ser distintas, así como el nivel de ruido. Sin embargo, los métodos para controlarlos son idénticos. A continuación, se señalan recomendaciones para el diseño del ambiente acústico:

1. En primer lugar, hay que determinar si el nivel de ruido es tal que provoca pérdida de la audición. Para ello, hay que comparar las medidas efectuadas con normas establecidas, donde se indican el nivel de ruido por actividad y los tiempos de exposición, asociados en tanto se tomen las medidas correctivas para reducir niveles de ruido perjudiciales (ver Cuadro 2).

2. Se conoce que un nivel sonoro por debajo de 85 $\mathrm{db}(\mathrm{A})$ no causa daño al trabajador. Sin embargo, puede ser muy molesto, dependiendo de la actividad que se desarrolla. A continuación, el Cuadro
3 muestra niveles de ruido recomendables para ambientes diversos.

3. Si se establece que el ruido es generado por una máquina, se debe ver la forma de modificarla, de manera que produzca menos ruido. A veces con bajar su velocidad es suficiente. También se puede efectuar un cerramiento total de la máquina o bien se puede optar por cambiarla por otra más silenciosa.

4. Si luego de aplicar la recomendación anterior, la incomodidad por el ruido persiste, se debe poner a disposición de los trabajadores equipos de protección individual como orejeras y tapones para oídos, sin descuidar la labor que se debe realizar para lograr que el trabajador use el equipo por propio convencimiento, motivando y supervisando su uso, principalmente cuando el nivel de ruido podría lesionar, con el tiempo, su capacidad auditiva.

5. Si las paredes del recinto son duras acústicamente, se producirán ecos y reflexiones, lo que incrementará la presión sonora. En este caso, se deben buscar materiales absorbentes para el revestimiento de las paredes, sobre todo, si hay máquinas ubicadas próximas a estas paredes. De esta forma, se reducirá la presión sonora.

6. La vibración producida por las máquinas también produce energía acústica, por lo que hay que hacer desde un análisis en la zona de su emplazamiento, colocando aisladores de vibración, hasta la verificación de piezas que pudieran estar sueltas o sin base firme.

Hay niveles sonoros que, sin dañar la salud del trabajador, producen una sensación de incomodidad. Esto depende mucho de cómo el oyente percibe el ruido y su contexto, más que el ruido en sí. Por lo tanto, hay que considerar situaciones como las que seguiremos describiendo para diseñar un ambiente acústico adecuado.

7. El ruido generalmente se asocia a la insatisfacción con el medio ambiente. Por ejemplo, en el trabajo de oficina, donde predominan las actividades mentales, si el nivel de ruido es muy alto, resulta difícil hablar por teléfono o mantener una comunicación oral, lo que se interpreta como una molestia provocada por el medio ambiente. Por otra parte, si el nivel de ruido es muy bajo, resulta muy perturbador el timbre inesperado de un teléfono. Por estas razones, se sugiere introducir lo que se llama un ruido blanco, para reducir la intensidad sonora, con respecto al ruido de fondo. 
Cuadro 2. Exposición máxima diaria a los niveles de ruido permitida por la Administración para la Salud y la Seguridad en el Trabajo de los Estados Unidos

\begin{tabular}{|l|c|c|c|c|c|c|c|}
\hline $\begin{array}{l}\text { Nivel de Ruido } \\
\text { en dB(A) }\end{array}$ & 85 & 90 & 95 & 100 & 105 & 110 & 115 \\
\hline $\begin{array}{l}\text { Exposición } \\
\text { máxima por día }\end{array}$ & $\begin{array}{c}16 \\
\mathrm{Hrs}\end{array}$ & $8 \mathrm{Hr}$ & $4 \mathrm{Hrs}$ & $2 \mathrm{Hrs}$ & $1 \mathrm{Hr}$ & $0.5 \mathrm{Hr}$ & $\begin{array}{c}0.25 \\
\mathrm{Hr}\end{array}$ \\
\hline
\end{tabular}

Cuadro 3. Niveles de ruido recomendados para ambientes diversos

\begin{tabular}{|ll|c|}
\hline \multicolumn{1}{|c|}{ Tipo de Recinto } & $\begin{array}{c}\text { Criterio de } \\
\text { ruido en dB(A) }\end{array}$ \\
\hline$\checkmark$ & $\begin{array}{l}\text { Grandes oficinas, almacenes, sala de } \\
\text { espera, restaurantes silenciosos }\end{array}$ & 35 \\
\hline$\checkmark$ & $\begin{array}{l}\text { Grandes restaurantes, oficinas con } \\
\text { máquinas de escribir }\end{array}$ & 45 \\
\hline$\checkmark$ & Grandes recintos con máquinas de oficina & 55 \\
\hline$\checkmark$ & Talleres & $45-75$ \\
\hline
\end{tabular}

8. La música en el trabajo puede ser considerada una ayuda en tareas repetitivas, monótonas y rutinarias o de esfuerzo físico, donde una sensación de comodidad y satisfacción podrían influir para el aumento de la vigilancia y del esfuerzo físico sostenido. No es recomendable cuando se requiera cierto nivel de concentración y, en todo caso, no debe constituir un elemento perturbador.

9. No sólo perturba la interferencia directa del ruido, sino también la indirecta. Por ejemplo, una oficina que se ve afectada por el nivel de ruido externo añade un componente de molestia adicional, al tener que mantener las ventanas cerradas en verano. En este caso, superado el problema acústico habría que considerar el problema de la temperatura ambiental.

\section{CONCIUSIONES}

La consideración más importante en el diseño de un ambiente acústico es la protección de la audición de los trabajadores. El ruido y la vibración de las máquinas deben ser minimizados, reemplazados o aislados del área de trabajo. Un enfoque ergonómico del ambiente sonoro debe ir más allá de la medida del ruido y debe considerar la comodidad acústica del personal.

El trabajo intelectual y creativo sufre mayores molestias por el ruido que por el trabajo rutinario. Sin embargo, puede decirse que depende, en gran medida, del sujeto receptor, razones que hay que tener en cuenta cuando se quiere poner música como parte del ambiente de trabajo.

\section{BIBLIOGRAFÍA}

1. Kong, Stephan. (1992). Diseño de Sistemas de Trabajo. Editorial Limusa. México.

2. Mondelo, Pedro R.; Gregori T., Enrique y Barrau B., Pedro. (2000). Ergonomía 1: Fundamentos. 3era Edición. Editorial AlfaOmega. México.

3. Ochoa Pérez, Juan y Bolaños, Fernando. (1990). Medida y control del ruido. Editorial Marcombo SA. España.

4. Ruiz Martínez, José Ramón. (2000). ¿Qué es el ruido?: Ergonomía. Departamento de Economía, Hacienda y Empleo del Gobierno de Aragón. España. 


\section{PROCEDIMIENTOUNIFICADO PARA BALANCEAR LAS REACCIONES REDOX EMPLEANDO EL MÉTODO DEL lON-ELECTRÓN}

(1) Mooner Lavado Soto

(2) Julio Yenque Dedios

\section{RESUMEN}

Las reacciones de oxidación-reducción, generalmente conocidas como reacciones redox, constituyen un grupo de reacciones químicas importantes. En el presente trabajo se propone un procedimiento unificado para balancear dichas reacciones, empleando el método del ion-electrón, ya sea en un medio ácido ó básico. Por lo general, el balanceo se realiza a través de diversas etapas durante gran parte de su ejecución.

Palabras Claves: Procedimiento unificado, reacciones Redox, método del ion electrón.

UNIFIED PROCEDURE FOR BALANCING THE Redox REACtion Through THE IONELECTRON METHOD

\section{ABSTRACT}

The oxidation-reduction reactions, typically known as "redox reactions", are the most important group of chemical reactions. This study proposes a unified procedure for balancing those reactions through an ionelectron method, either in acid or basic medium. Traditionally, balancing is carried out through different stages in most parts of the process.

Key words: Unified procedure, Redox reactions, Ion-electron method.

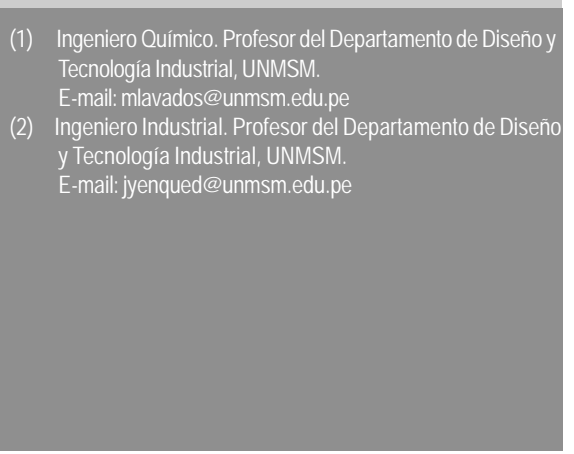

\section{INTRODUCCIÓN}

Las reacciones de oxidación-reducción, comúnmente denominadas reacciones redox, son reacciones de transferencia de electrones, en las que las especies químicas que pierden uno o más electrones se oxidan y, por el contrario, las que ganan uno o más electrones se reducen. El método del ion-electrón se fundamenta en el uso de iones hidrógeno $\left(\mathrm{H}_{+}\right)$, iones hidróxido $(\mathrm{OH}-)$ y electrones (e-) durante el proceso de balanceo de átomos y cargas en las medias reacciones, en las que la reacción total suele desdoblarse. El medio en el que se producen estas reacciones puede ser ácido o básico.

\section{ETAPAS DEL PROCEDIMIENTO UNIFICADO}

Para explicar el procedimiento se utilizan las especies hipotéticas $\mathrm{R}_{3} \mathrm{O}_{4}$ y $\mathrm{MO}_{4}^{-}$como reactivos, siendo los productos $\mathrm{R}_{2} \mathrm{O}_{3}$ y $\mathrm{M}^{2+}$.

\section{Medio ácido}

El medio ácido se caracteriza por la presencia de iones hidrógeno $\left(\mathrm{H}^{+}\right)$en disolución acuosa.

El procedimiento consta de las siguientes etapas:

1. Escritura de la reacción en forma iónica.

$$
\mathrm{R}_{3} \mathrm{O}_{4}+\mathrm{MO}_{4}^{-}+\mathrm{H}^{+} \longrightarrow \mathrm{R}_{2} \mathrm{O}_{3}+\mathrm{M}^{2+}
$$

2. Desdoblamiento de la reacción en dos medias reacciones o semireacciones: Una de oxidación y otra de reducción.

$$
\begin{aligned}
& \mathrm{R}_{3} \mathrm{O}_{4} \longrightarrow \mathrm{R}_{2} \mathrm{O}_{3} \\
& \mathrm{MO}_{4} \longrightarrow \mathrm{M}^{2+}
\end{aligned}
$$

3. Balanceo de átomos, introduciendo agua $\left(\mathrm{H}_{2} \mathrm{O}\right)$ con el fin de balancear los oxígenos e iones hidrógeno para los hidrógenos presentes en el agua.

$$
\begin{aligned}
& \mathrm{H}_{2} \mathrm{O}+2 \mathrm{R}_{3} \mathrm{O}_{4} \longrightarrow 3 \mathrm{R}_{2} \mathrm{O}_{3}+2 \mathrm{H}^{+} \\
& 8 \mathrm{H}^{+}+\mathrm{MO}_{4}^{-} \longrightarrow \mathrm{M}^{2+}+4 \mathrm{H}_{2} \mathrm{O}
\end{aligned}
$$


4. Balanceo de cargas, introduciendo el número necesario de electrones.

$$
\begin{aligned}
& \mathrm{H}_{2} \mathrm{O}+2 \mathrm{R}_{3} \mathrm{O}_{4} \longrightarrow 3 \mathrm{R}_{2} \mathrm{O}_{3}+2 \mathrm{H}^{+}+2 \mathrm{e}- \\
& 8 \mathrm{H}^{+}+\mathrm{MO}_{4}{ }^{-}+5 \mathrm{e}^{-} \longrightarrow \mathrm{M}^{2+}+4 \mathrm{H}_{2} \mathrm{O}
\end{aligned}
$$

5. Igualación del número de electrones en cada semireacción, para lo cual estas se multiplican por un coeficiente mínimo que permita la igualdad. En este caso, los coeficientes son 5 y 2.

$$
\begin{aligned}
& 5 \mathrm{H}_{2} \mathrm{O}+10 \mathrm{R}_{3} \mathrm{O}_{4} \longrightarrow 15 \mathrm{R}_{2} \mathrm{O}_{3}+10 \mathrm{H}^{+}+10 \mathrm{e}- \\
& 16 \mathrm{H}^{+}+2 \mathrm{MO}_{4}^{-}+10 \mathrm{e}-\longrightarrow 2 \mathrm{M}^{2+}+8 \mathrm{H}_{2} \mathrm{O}
\end{aligned}
$$

6. Suma de las dos semireacciones para obtener la reacción total. Se observa que, al efectuar la suma, los electrones desaparecen.

$$
10 \mathrm{R}_{3} \mathrm{O}_{4}+2 \mathrm{MO}_{4}^{-}+6 \mathrm{H}^{+} \longrightarrow 15 \mathrm{R}_{2} \mathrm{O}_{3}+2 \mathrm{M}^{2+}+3 \mathrm{H}_{2} \mathrm{O}
$$

\section{Medio básico}

La presencia de los iones hidróxido $\left(\mathrm{OH}^{-}\right)$es una característica de un medio básico. Tomando en cuenta el mismo ejemplo, el procedimiento unificado es común hasta la etapa 6 . A partir de allí, se introduce, en ambos lados de la reacción total, un número de iones hidróxido igual al de los iones hidrógeno presentes.

$$
10 \mathrm{R}_{3} \mathrm{O}_{4}+2 \mathrm{MO}_{4}^{-}+6 \mathrm{H}^{+}+60 \mathrm{H}^{-} \longrightarrow 15 \mathrm{R}_{2} \mathrm{O}_{3}+2 \mathrm{M}^{2+}+3 \mathrm{H}_{2} \mathrm{O}+60 \mathrm{H}^{-}
$$

Seguidamente, los iones $\mathrm{H}^{+}$y OH , presentes en un mismo lado de la reacción, se integran como $\mathrm{H}_{2} \mathrm{O}$.

$10 \mathrm{R}_{3} \mathrm{O}_{4}+2 \mathrm{MO}_{4}^{-}+6 \mathrm{H}_{2} \mathrm{O} \longrightarrow 15 \mathrm{R}_{2} \mathrm{O}_{3}+2 \mathrm{M}^{2+}+3 \mathrm{H}_{2} \mathrm{O}+60 \mathrm{H}^{-}$

Finalmente, ajustando el $\mathrm{H}_{2} \mathrm{O}$ se tiene:

$10 \mathrm{R}_{3} \mathrm{O}_{4}+2 \mathrm{MO}_{4}^{-}+3 \mathrm{H}_{2} \mathrm{O} \longrightarrow 15 \mathrm{R}_{2} \mathrm{O}_{3}+2 \mathrm{M}^{2+}+60 \mathrm{H}^{-}$

\section{CONCLUSIONES}

Las seis etapas del procedimiento unificado son comunes. Asimismo, el paso al medio básico consiste en añadir a la reacción total obtenida, utilizando el medio ácido, un número de iones $\mathrm{OH}^{-}$que neutralicen a los iones $\mathrm{H}^{+}$existentes.

\section{BIBLIOGRAFÍA}

1. Chang, Raymond y College, Williams. (2003). Química. Ed. Mc Graw Hill. México.

2. Lavado, Mooner. (2005). Química General. Edición inédita (en revisión). Lima, Perú. 